\title{
Primary Adenocarcinoma of the Fallopian Tube
}

\author{
${ }^{1}$ Narayana S wamy M, ${ }^{2}$ Manjunath RL, ${ }^{3}$ Udaya Kumar M, ${ }^{4}$ Kalyani $R,{ }^{5}$ Avinash Patil, ${ }^{5}$ Shobithe J ain \\ ${ }^{1}$ Professor and Head, Department of Obstetrics and Gynecology, Sri Devaraj URS Academy of Higher Education and \\ Research Center, Kolar, Karnataka, India \\ ${ }^{2}$ P rofessor, Department of Obstetrics and Gynecology, MS Rammaiah Medical College, Bengaluru, Karnataka, India \\ ${ }^{3}$ Associate Professor, Department of Pathology, Sri Devaraj URS Academy of Higher Education and Research Center \\ Kolar, Karnataka, India \\ ${ }^{4}$ P rofessor, Department of Pathology, Sri Devaraj URS Academy of Higher Education and Research Center \\ Kolar, Karnataka, India \\ ${ }^{5}$ Resident, Sri Devaraj URS Academy of Higher Education and Research Center, Kolar, Karnataka, India
}

Correspondence: M Narayana Swamy, Professor and Head, Department of Obstetrics and Gynecology, Prakruthi, 254 1st Cross, 1st Main, New Extension, Near Doomlight Circle, Kolar-563101, Karnataka, India, Phone: 09845437623 e-mail: drnswamy12@ rediffmail.com

\section{ABSTRACT}

Carcinoma of the fallopian tube is not one of the commonest cancer encountered in the clinical practice. Nevertheless, it is vital to have clear knowledge about this disease, since it often presents with various common disorders and when missed, it lends poor prognosis to the patient.

Keywords: Carcinoma, Fallopian tube chemotherapy.

\section{INTRODUCTION}

Primary tumors of the fallopian tube are rare, accounting for only 0.31 to $1 \%$ of all gynecological malignancies.' Primary fallopian tube carcinoma occurs in the age ranging from 18 to 88 years with the usual age of occurrence between 40 to 65 years and a mean age of 55 years. Even though the etiology is not known, it is reported to be associated with chronic tubal inflammation, infertility, tuberculous salpingitis, and tubal endometriosis. ${ }^{2}$ Preoperative diagnosis of this malignancy is difficult due to its insidious onset and silent course, and the diagnosis is usually made at laparotomy or by postoperative histology. ${ }^{3}$

\section{CASE REPORT}

A 40-year-old M uslim woman presented with complaints of foul smelling vaginal discharge since one month, and lower abdominal pain since 4 days. Her menstrual cycles were regular with last menstrual period 8 days ago. She was para 2 with last childbirth and tubal ligation 8 years ago. Her mother had died cervical cancer. She was obese with weight of $75 \mathrm{~kg}$. Physical and systemic examinations were normal. Genital examination reveal ed hypertrophied cervix and bulky uterus with nontender fullness of 7 to $8 \mathrm{~cm}$ in the right fornix.

$\mathrm{Her}$ hepatic, renal function, and the chest radiograph were normal. HIV I, II, and serum A ustralia antigen were negative. Pap smear showed inflammatory picture. UItrasonography showed a bulky uterus with right tubo-ovarian mass measuring of $7.8 \times 4 \mathrm{~cm}$. She was diabetic and anemic, which were treated preoperatively.

Patient underwent laparotomy on 16 J uly 2003. There was a $6 \times 5 \mathrm{~cm}$ fleshy growth arising from the right fallopian tube.
Para-aortic lymph nodes were enlarged, matted and unresectable. She underwent total hysterectomy, bilateral sal pingo-oophorectemy and partial omentectomy. A scitic fluid was positive for malignant cells.

\section{PATHOLOGICAL EXAMINATION OF THE SPECIMEN}

U terus was bulky and measured $10 \times 8 \times 5 \mathrm{~cm}$ with a grey-white tumor nodule in the myometrium. The right tube was diffusely enlarged measuring $5 \times 4 \times 3 \mathrm{~cm}$ with fimbrial extension. The right ovary measured $4 \times 2.5 \times 1.5 \mathrm{~cm}$. The cut surface showed a solid grey-white tumor (Fig. 1). The left ovary measured $4 \times$ $3 \times 1 \mathrm{~cm}$ with unremarkable cut surface.

The histological examination demonstrated features of moderately differentiated adenocarcinoma with transmural infiltration of the right fallopian tube (Figs 2 and 3 ). The right ovary, left ovary and the myometrium showed tumor deposits and vascular emboli. The left tube was normal and free from the tumor. The omentum showed deposits of this tumor. The endometrium and the endocervical canal were unremarkable.

Between July 30th 2003 and 8 January 2004, patient received six cycles of chemotherapy with injection. Paclitaxel $230 \mathrm{mg}\left(175 \mathrm{mg} / \mathrm{m}^{2}\right)$ and Carboplatin $450 \mathrm{mg}\left(300 \mathrm{mg} / \mathrm{m}^{2}\right)$ injections once in 4 weeks. On 9 A ugust 2004, patient was admitted with dysphagia, ascites and foul smelling ulceration of the inguinal regions.

Ultrasound showed mixed echogenic lesion of $5 \mathrm{~cm}$ in right adnexa, gross ascites, peritoneal thickening and omental cakes. She was treated with antibiotics for the secondary infection of the inguinal ulcers. Patient succumbed to the disease on 2 September 2004 due to multiple metastases. 


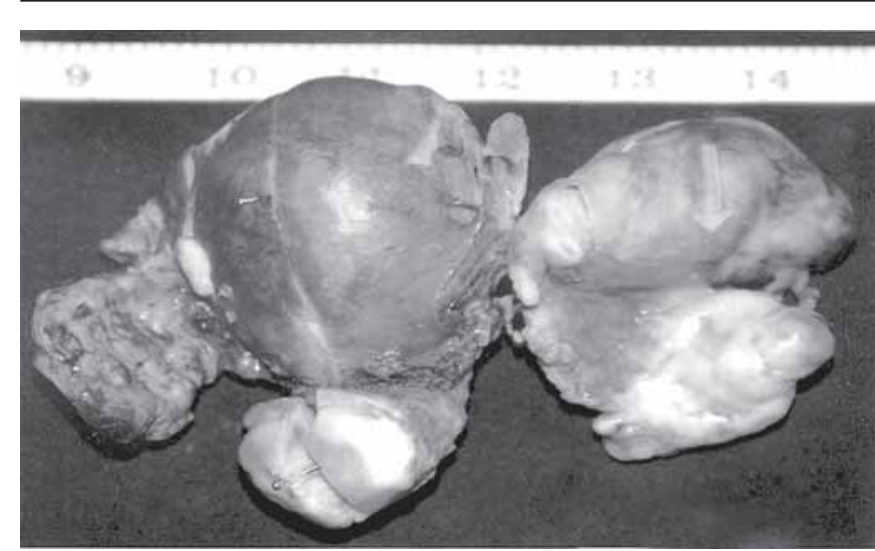

Fig. 1: Gross photograph showing diffuse tubal enlargement with tiny grey-white serosal deposits

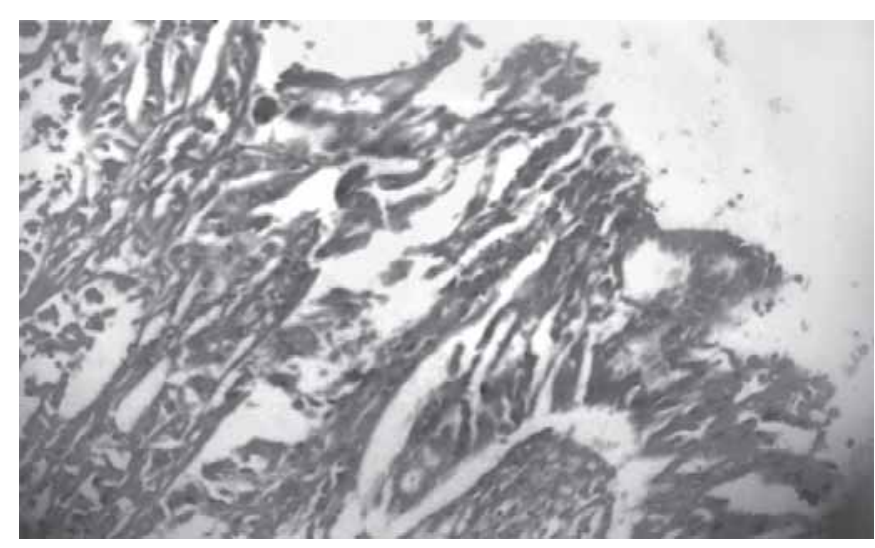

Fig. 2: Microphotograph showing tubal mucosal adenocarcinoma $(\mathrm{H}$ and $\mathrm{E} \times 400)$

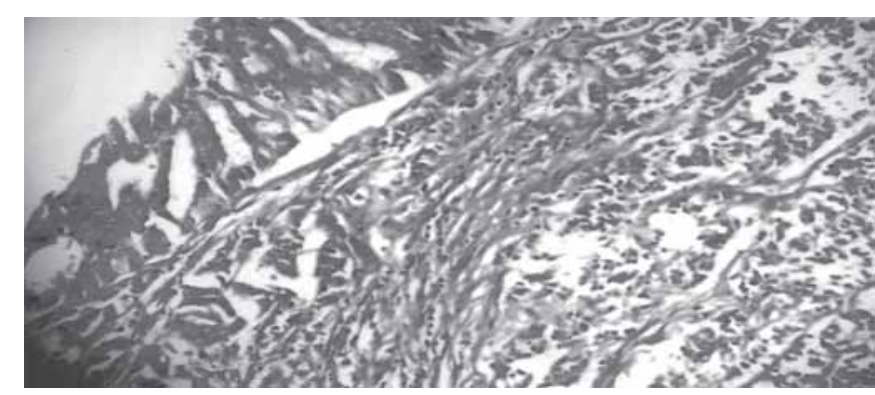

Fig. 3: Microphotograph with mucosal and mural infiltration of adenocarcinoma ( $\mathrm{H}$ and $\mathrm{E} \times 400)$

\section{DISCUSSION}

Primary carcinoma of the fallopian tube is rare but aggressive tumor. The mean age of incidence is 55 years. The presenting symptoms are variable. In more than half of the cases, women present with vaginal bleeding. The classic triad of symptomsvaginal bleeding, pelvic pain, and watery vaginal discharge are seen infrequently. A pelvic mass is the most common physical finding. ${ }^{4} \mathrm{M}$ ost cases are preoperatively diagnosed to have ovarian carcinoma, uterine fibroid or pelvic inflammatory disease. Tumor marker CA-125 may be elevated in cases of tubal cancer. The FIGO staging system (1991) of tubal carcinoma follows the general outline of ovarian carcinoma as the pattern of spread, the anatomic location of ovarian, and tubal carcinomas appear to be similar. Primary route of spread of fallopian tube carcinoma is by lymphatics. As the tube is rich in lymphatics, it facilitates the spread of tumor once the mucosa is breached. The other methods of spread are: direct extension and transcelomic spread. Surgery is the main stay of treatment of carcinoma of fallopian tube. The current recommendation is to perform total abdominal hysterectomy, bilateral salpingo-oophorectomy, appendicectomy, omentectomy, and retroperitoneal lymph node sampling. ${ }^{5}$

Patients with advanced (stage II and III) fallopian tube carcinoma with optimal debulking will benefit from whole abdomen radiation with pelvic boost. A dvanced disease with unresectable para-aortic lymph nodes is the main hindrance to curative treatment. ${ }^{6}$

Our patient had stage III tubal malignancy with large unresectable para-aortic lymph nodes. She had residual/ recurrent disease and succumbed to it in 14 months.

\section{REFERENCES}

1. K huspreet W alia, U sha B andlish, R uby B hatia, Seema Sharma. Fallopian tube carcinoma: A rare cause of postmenopausal bleeding. Obs and Gynae Today 2000;5:193-94.

2. Ritalovine Demopoulos, Rima A ronov, A ugusto M eisa. Clues to the pathogenesis of fallopian tube carcinoma: A morphological and immunological case control study. Int J Gynecol Pathol 2001;20:128-32.

3. Benjamin R, Sheard J, kabyemela J, Tagore V , K irwan J. First report of primary fallopian tube cancer diagnosed by liquid based cytology in an asymptomatic woman. BJOG 2007;114: 1575-76.

4. David P Warshal, Elise R B urgelson, James K Aikins, Thomas $F$ Rocereto. Post hysterectomy fallopian tube carcinoma presenting with a positive papanicolaou smear. Obstet Gynecol 2000;95:834-35.

5. Ajith kumar TV, M inimole A L, M anju M ary J ohn, A shok K umar OS. Primary fallopian tube carcinoma. Obstet Gynecol Surv 2005;60(4):247-52.

6. Angiolo Gadducci. Current management of fallopian tube carcinoma. Current Opinion in Obstetrics and Gynecology 2002;14:27-32. 